\title{
CLOUD AGNOSTIC STREAM ANALYTICS
}

\author{
Chandan Kumar \\ Ryerson University, \\ beCloudready, Toronto, Canada
}

\begin{abstract}
As machine learning is getting more and more mainstream, increasing number of public cloud providers are providing machine learning related APIs such as Text Analytics, ANN, Auto ML (pretrained) APIs which are on one hand make life easier for many application developers, but on the other hand brings API management complexity in hybrid or multi cloud environment.

The current evolution of these ML (machine learning) APIs leads to more coherent integration with the current cloud provider and makes it harder to switch between the providers. These leads to a vendor locked in situation where it becomes difficult for application to easily switch the service provider.

A cloud agnostic framework has been proposed here which would allow to develop modern cloud-based data science, machine learning, AI applications using the proposed framework or similar approaches where the application provider could seamlessly switch between various cloud vendors as well as native OS level APIs painlessly and programmatically. As the subject of data science is so broad that we will focus only on one aspect of it to show case the proof of concept of the framework using sentiment analysis APIs to demonstrate stream analytics capabilities. Same approach could easily be applied on other APIs as well.
\end{abstract}

\section{KEYWORDS}

Bigdata, Stream Analytics, Cloud Agnostic, Sentiment Analytics

\section{INTRODUCTION}

There are two aspects need to be addressed, when it comes to building stream analytics data pipeline. One being the data science part (building the model, Algorithm), other being the data engineering part, which involves preparing the data and deploying it in production environment. Often deploying in production environment is time consuming, expensive and difficult to maintain due to the fact that deploying a good model into production has IT related complexity such as security, compliance, high availability and performance.

In this paper we are trying to solve both problems from the business use case and application standpoint rather than from a data scientist standpoint, which mean this paper will focus on effectively utilizing available solution to help rapid application development rather than creating brand new model we are doing so by using cloud native APIs.

We will be using Python as application development language. Although, Python has inbuild support for NPL and used heavily among the Python community members, the availability of cheap cloud APIs has made it very attractive and easy to use state of the art Algorithms when it comes to any kind of machine learning or sentiment analysis.

As already mentioned, we will be using publicly available social media data (tweets) to build a cloud agnostic sentiment analytics platform (stream analytics platform). As with any other data pipeline, before we could even use these libraries, the biggest challenge will be to parse the tweets and make sure they are ready to be consumed by the API. Few limitations are very common, especially when it comes to sentiment Analysis such as Language issues. Most sentiment analysis libraries only support small percentage of languages notably

In this paper we will be using sentiment analysis use case for demonstrating the stream analytics capabilities using Cloud (SaaS) APIs. The major application of sentiment analysis is applicable to product reviews, political opinions, movie reviews, and even health related trends. 
As we are considering streaming the data rather than storing and analyzing it later, we have to do the data preparation on the fly (Huggani et al., 2014). Fortunately, Python has rich set of string manipulation libraries which could help us with these issues. There are plenty of sentiment analysis APIs and libraries available; however, later Cloud that provided APIs have become norm across multiple application to get the best possible outcome. I will be evaluating the following algorithms;

- Google Natural Language API

- Azure Text Analytics API

- AWS Comprehend

Hence our front end will be only a wrapper script which could either run on local machine or on the EC2 or even in AWS Lambda.

\section{LITERATURE REVIEW}

Vast majority of stream analytics-based research are done on IoT application, which involves stream of data being generated by sensors and which are analyzed on the edge device for certain time window to make quick prediction or trigger some alarm (Sikder et al., 2018). Some researchers such as Jayaraman et al., (2017) are also focused on building a multi-cloud analytics as service concept on semantically enabled hierarchical processing. Ari et al., (2014) implemented Pearson-Product Moment Correlation for analytics and Apriori \& FPGrowth algorithms for stream mining inside a popular event stream processing engine called Espe, in which they used both correlation analysis and association rule mining (ARM) over streams. In this context, they used cloud as source of the data rather than destination. Cloud based solution often provides an alternative with no capital requirement where lot of Machine learning APIs are available as service. Huqqani et al., (2014) articulates that various cloud-based solution providers are able to provide cost efficient GPU based solution which outperforms traditional GPU based solution when it comes to performance. In the experiment they explained how various parallel execution on large data set could be used GPU based Cloud solution to achieve higher performance for neural networks. Twitter is a stream-based application where you get instantaneous information, hence the data need to be analyzed on the fly. Researchers in Japan developed real-time earthquake-monitoring systems based on Twitter using streaming API to generate live alerts (Ari et al., 2012). Yang (2017), articulated how streaming solution for IoT devices could be used in large dataset to investigate fog system in certain geographical region for monitoring and alerting system. Nair et al. (2018), introduced a real-time health status prediction system and the user communicate via Twitter. The Twitter data was used as input their Spark base ML libraries to create a predictive system (Sakaki et al., 2010).

\section{DESIGN ARCHITECTURE}

A text mining task has always been one of the challenging tasks in the field of data analytics simply because of unpredictability of the data source. The data source could come from hundreds of languages which possess another challenge to most of the NPL algorithms as most of them are designed and optimized for English language. Often non-Latin languages possess another challenge in terms of UTF encoding, where if the programmer is not careful, it can easily crash the application.

\subsection{Data Ingestion}

- Data ingestion is the key to any type of data pipeline or data warehousing solution. Well defined data ingestion method and strategy goes long way to ensure the right data is present in the system for required analytics work. In current implementation data ingestion is very simple which means pulling the tweets from tweeter rest API, but we will ensure that a common API is developed so that in future more data sources could be easily added. 


\subsection{Data Wrangling}

- The concept and importance of data wrangling could not be better explained then the research paper done by Kandel et al., (2011). Later on, Kandel and Heer went on to create in 2012 a data wrangling company called Trifacta

- Data Wrangling mean preparing the data to be ready for analytics work. Simple example could be cleaning up the data by removing stem words, removing not supported languages or anything that deems not necessary for analytics purpose.

- Language detection (Python or Azure)

\subsection{Data Analytics}

Data analytics in case will involve two aspects.

- Sentiment Analysis.

- Aggregating the sentiment over time series.

Part one will be done by Cloud APIs such as Google Cloud NPL, AWS Comprehend and Azure Text Analytics. The sentiment would be recorded for each and every tweet and will be stored into a data frame/table. We can use either data frame or table in a SQL database to tabulate the time series data and to measure various descriptive statistical associated with the sentiment polarity and magnitude, so analyze what is the overall picture for the topic.

\section{APPROACH}

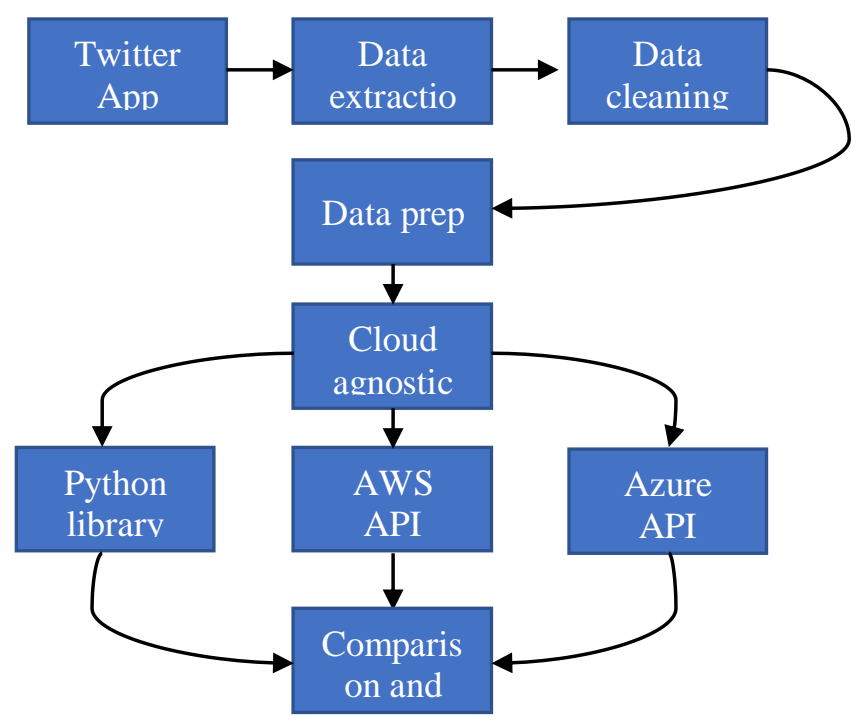

Figure 1. The data pipeline approach used for the application 


\subsection{Cloud Agnostic API Wrapper}

One of the key goals of this project is to have a seamless integration and access to most, if not all, AI APIs in a unified, simplified and cost-efficient manner. While doing so, the project will try to provide a solution where users can avoid all pitfalls of being vendor locked-in. Lately getting vendor locked-in has become a concern among various enterprise. If you deploy your infrastructure in one vendor's public cloud, say Amazon AWS, what will you do if the service fails to meet your standards or if the prices increase? What if the features you need are no longer supported? Sure, there are a couple of other big public Cloud providers - Microsoft Azure and Google Cloud. More vendors are making sure that their technologies are supported across multiple public Clouds. But it can be complicated and time consuming to move your applications and data from one Cloud to another.

The software has been written in a modular fashion [Figure 1] by keeping each service provider, whether native Python libraries or any public cloud APIs. The data structure used to prepare the data, as discussed in data cleaning and data prep section, is Python Pandas data frame.

The project layout shown in the image above depicts the class layout of the application. At the current state of the project it only supports following providers

- AWS

- Azure

- Python text blob

Similarly, more providers could be incorporated.

\section{APPLICATIONS}

\subsection{Small Business, Retail Customers}

At the moment, getting access to good quality analytics tool is often expensive, time consuming, and in most cases out of reach for these businesses. This tool could leverage the free tier service provided by most of the Cloud service providers, which would allow them to have access to top quality analytics tool at reasonable price or even free by spreading the quarries across the various providers using the Cloud agnostic API wrapper.

\subsection{Enterprises looking for a Cloud Agnostic AI API Service}

Enterprises adoption to AI and analytics services have increased dramatically; however, their IT infrastructure to support such massive change has not grown to the same pace. On the other hand, onboarding cost of on-prem AI infrastructure and team is very costly and time consuming. This tool can help them to achieve vendor agnostic interface and implementation to their services and hence providing better flexibility.

\subsection{Ability to choose the Best Model for Select Data Sets}

With array of algorisms and cloud service providers, it is becoming increasingly difficult to identify which pre-trained model works best for certain data sets. This tool could help developers to archive some level of comparison of different libraries against the same data set.

\section{REFERENCES}

Ari, I., Olmezogullari, E., and Çelebi, O.F. (2012). Data stream analytics and mining in the cloud. 4th IEEE International Conference on Cloud Computing Technology and Science Proceedings, Taipei. p. 857-862. doi: 10.1109/CloudCom.2012.6427563

Huqqani, A. A., Schikuta, E., \& Mann, E. (2014). Parallelized neural networks as a service. 2014 International Joint Conference on Neural Networks (IJCNN), 2282-2289. doi:10.1109/IJCNN.2014.6889742 
Jayaraman, P. P., Perera, C., Georgakopoulos, D., Dustdar, S., Thakker, D., and Ranjan, R. (2017) Analytics-as-a-service in a multi-cloud environment through semantically enabled hierarchical data processing. Softw. Pract. Exper., 47: 1139- 1156. doi: 10.1002/spe.2432.

Kandel, S., Heer, J., Plaisant, C., Kennedy, J., Frank van Ham, Riche, N.H., Weaver, C., Lee, B., Brodbeck, D., Buono, P., (2011) Research directions in data wrangling: visualizations and transformations for usable and credible data. Information Visualization, 10 (4), p.271-288. doi: 10.1177/1473871611415994.

Nair, L. R., Shetty, S. D., \& Shetty, S. D. (2018). Applying spark-based machine learning model on streaming big data for health status prediction. Computers and Electrical Engineering, 65, 393-399.

Sakaki, T., Okazaki, M., and Matsuo. Y. (2010). Earthquake shakes Twitter users: real-time event detection by social sensors. Proceedings of the 19th international conference on World wide web. ACM, p. 851-860.

Sikder, A. K., Petracca, G., Aksu, H., Jaeger, T. and Uluagac, A. S. (2018). A survey on sensor-based threats to internet-of-things (IoT) devices and applications. arXiv preprint arXiv:1802.02041.

Yang, S. (2017). IoT stream processing and analytics in the fog. IEEE Communications Magazine, 55(8), 21-27. doi:10.1109/MCOM.2017.1600840 